\title{
Reduction Of Energy Losses In The End wall Junction Area Through The Addition Of Forward Facing Step Turbulent Generator
}

\author{
Mirmanto, $\mathrm{H}^{1 \mathrm{a} a}$, Sutrisno ${ }^{2, \mathrm{~b}}$, Sasongko, H. ${ }^{1, \mathrm{c}}$, Noor, D.Z. ${ }^{1, \mathrm{~d}}$ \\ ${ }^{1}$ Institute of Technology Sepuluh Nopember,Surabaya, East Java, Indonesian \\ ${ }^{2}$ Petra Christian University, Surabaya, East Java, Indonesian

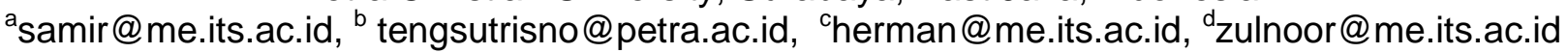

Keyword : Secondary flow, forward facing step, turbulent intensity.

\begin{abstract}
The research is conducted in order to reduce energy losses caused by the secondary flow in the endwall junction. This phenomenon is caused by the interaction of two adjacent viscous flow (symmetric airfoil and endwall). Reduction of energy loss carried out by addition of Foward Facing Step Turbulator (FFST) in the upstream. Endwall junction area is modeled as a NACA 0015 airfoil and a flat plate. Position of FFST is at a distance $\mathrm{L}=2 / 3 \mathrm{C}$ upstream leading edge and a thickness $d=4 \% \mathrm{C}$. Free stream conditions $\mathrm{Re}_{\mathrm{d}}=10^{5}$ with turbulence intensity $(\mathrm{Tu}) 5 \%$. Research is conducted by numerical and experiment methods. Pathlines of numerical result methods has an identic structure with "Oil Flow Visualization" of the experiment.

Result of the research states that the addition of FFST can increase the turbulence intensity in the flow near the wall. So at the same angle of attact $(\alpha)$, the saddle point position on the leading edge has distance nearly the same but a little more towards the lower side and the separation line is wider than without FFST. Because the flow has stronger turbulence intensity, attachment line of the upper and lower sides have a better capability of following the contours of the body. So the point of separation can be delayed and blockage (energy loss) can be reduced as well. Reduction of energy loss is most effective on $\alpha=8^{\circ}(4.16 \%)$,
\end{abstract}

\section{Introduction}

Secondary flow is a flow that has a velocity component in the orthogonal direction to the main flow. The flow is caused by the interaction of two viscous flow across the body and adjacent end wall. On the application, this phenomenon is often found in the interaction of wing and fuselage aircraft, rotor and hub in axial compressors, etc. Due of the influence of secondary flow, blockage and loss of energy in the end wall junction occur. According Laksminaraya and Horlock [1] secondary flow effects on axial compressor causing the loss of $50 \%$ of the total hydraulic losses. Therefore research in order to reduction of energy losses due to secondary flow is still being developed. Research on secondary loss reduction has been performed by Mirmanto and Sasongko [2] by adding leading edge fairing on a single body of NACA 0020 and NACA 0015 . The results showed a decrease in the intensity of the vortex at the front of leading edge, so that the 3D separation line is closer to follow the contour body and corner wake (blockage) is formed on the upper side getting smaller. In general, the secondary flow caused by the 3D flow separation in the endwall junction due to incompetence fluid energy to overcome of the adverse pressure and friction effects. In the previous studies, only focus on body modification to reduce the adverse pressure. On the other side (boundary layer theory), Schlighting [3] states that the more turbulent flow of velocity profile has better condition to overcome the adverse pressure and friction effects. Thus some researchers try to improve the energy by adding a turbulent free stream generator (turbulator) in front of the body. Djijali [4] conducted numerical and experimental study at steady state flow and showed that the separation bubble causes an increase in the turbulence intensity and momentum near the end wall. Further experimental of Yaghoubi and Mahmoodi [5] and supported by simulation results Suksangpanomrung et al [6] stated that, separation bubble is found right after crossing the stream bluff rectangular turbulator (BRT), where the position of the reattachment point is determined by the dimensions of the BRT itself. Saldana et al [7] stated that BRT is placed on a flat plate surface track called Step Forward facing Turbulator (FFST). The flow characteristics have 
two point of separation in two parts. It is developed by Serry et al [8]. Applications use the forward facing step Turbulator (FFST) proved to produce a more turbulent flow. So the application of FFST in the upstream end wall junction is expected to reduce energy losses.

\section{Methodology}

The research was conducted numerically and experimentally. The body of airfoil is NACA 0015 (Mirmanto and Sasongko [2]). Skets of position and geometry FFST is shown in Fig 1. Distance $\mathrm{L}=2 / 3 \mathrm{C}$, distance FFST to the inlet free stream $(\mathrm{Lu}=8 / 15 \mathrm{C})$ and thickness $\mathrm{d}=4 \% \mathrm{C}$. Numerical simulations performed using flow free stream Red $=10^{5}$ with turbulence intensity $(\mathrm{Tu})$ $5 \%$.
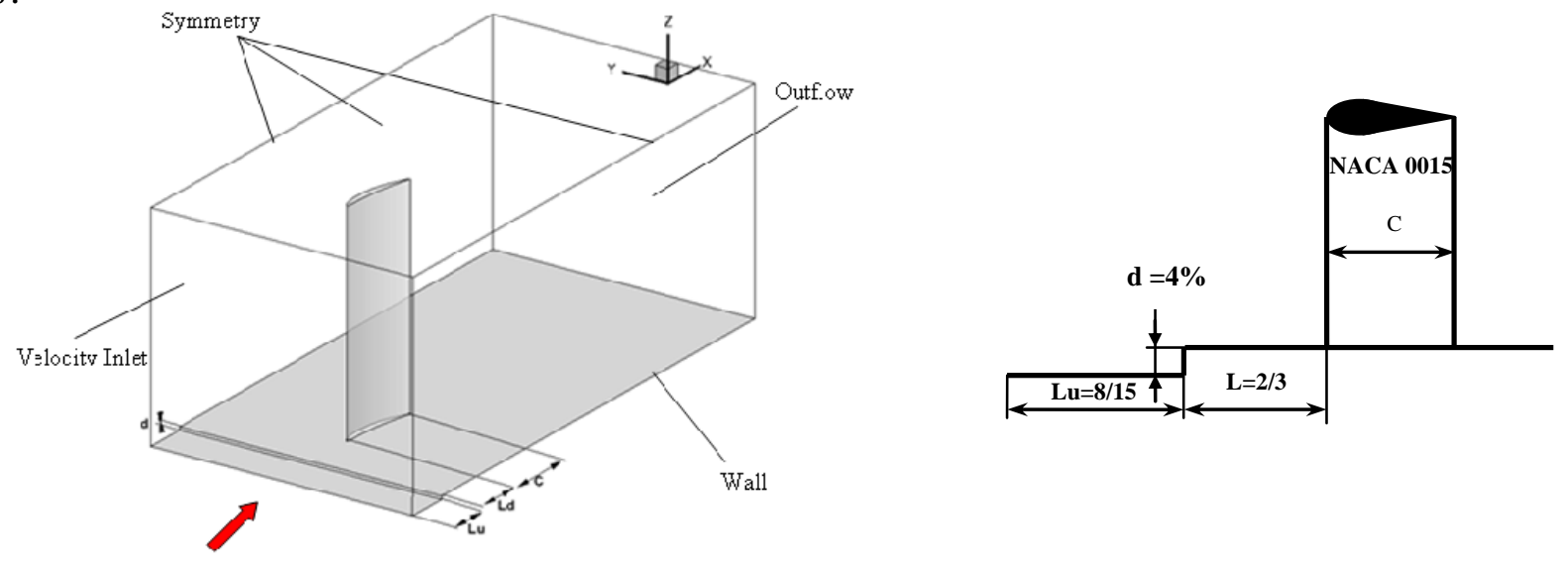

Flow Freestream

Fig. 1. Dimension of FFST

In the numerical modeling, grid independency is in accordance with recommendation of Jones and Clarke[9]. Velocity values obtained at position $\mathrm{x}=164 \mathrm{~mm}$ and $\mathrm{y}=0.5 \mathrm{~mm}$, shape factor (SF) derived from the velocity profile at position $\mathrm{x}=164$. Furthermore, the multiple meshing shown in Table 1. Mesh C and D show that the first node in $\neg$ near wall y + value 19952 so it can be concluded in the laminar sublayer. As for the Mesh A and B values of endwall be the first node in the laminar sublayer and the speed and shape factor values are almost the same. Thus the selected mesh which has a value of $\mathrm{y}+\mathrm{a}$ smaller mesh B. So mesh modeling $\mathrm{B}$ is chosen for the entire simulation.

Numerical Simulation in this study is carried out using commercial programs ANSYS FLUENT 14.5, where TECPLOT 3602010 is used in order to display the flow structure. The research is conducted with variations of Angle of Attack (AoA) between 0,4,8,12 and 16. The results of research will be validated by experiment results in the form of skin friction line on the endwall surface structure (OFV). Evaluation will also analyze kinetic velocity and contours of turbulent intensity on the surface of the body and the endwall. Energy losses are analyzed via contour of axial total pressure loss coefficient on downstream areas.

Table. 1 Evaluated Grid Independency.

\begin{tabular}{|c|r|r|r|r|r|r|c|}
\hline Model & \multicolumn{1}{|c|}{ Node } & \multicolumn{1}{c|}{ Cells } & \multicolumn{1}{c|}{$\mathrm{y}^{*}$} & \multicolumn{1}{c|}{$\mathrm{y}^{*}$} & \multicolumn{1}{c|}{ Iterasi } & $\begin{array}{c}\text { Velocity }(\mathrm{m} / \mathrm{s}) \\
(\mathrm{x}=164 \mathrm{~mm}, \mathrm{y}=0.5 \mathrm{~mm})\end{array}$ & $\begin{array}{c}\text { Shape Factor } \\
\mathrm{H}=\delta^{*} / \theta\end{array}$ \\
\hline Mesh A & 177,711 & 176,000 & 4.761 & 4.739 & 2102 & 9.53 & 2.02 \\
Mesh B & 116,497 & 115,200 & 7.772 & 8.904 & 2033 & 9.52 & 2.00 \\
Mesh C & 68,886 & 68,000 & 11.928 & 13.175 & 1047 & 8.97 & 2.09 \\
Mesh D & 34,386 & 33,800 & 19.952 & 21.731 & 710 & 8.48 & 2.66 \\
\hline
\end{tabular}

\section{Analysis And Result}

3D flow separation is begun by frontal collision attachment-line from inlet up stream with the attachment-line of the leading edge of the body. Interaction of two attachment-line will cause 
the 3D flow separation. Furthermore, the flow will met with the attachment-line coming from the side of the body and form a skewed boundary layer. Furthermore it will match with adverse pressure gradient in front of the leading edge, the flow will roll up and move towards the downstream.

At $\alpha=0^{0}$, the flow structures by additional of FFST, show that the end position of the stagnation point on the leading-edge has a more distance to end wall because the flow has a disturbance thickness or boundary layer is thicker and stronger turbulent intensity. Fig. 2 shows that velocity profile and turbulent intensity difference between the flat plate (without FFST and with FFST).
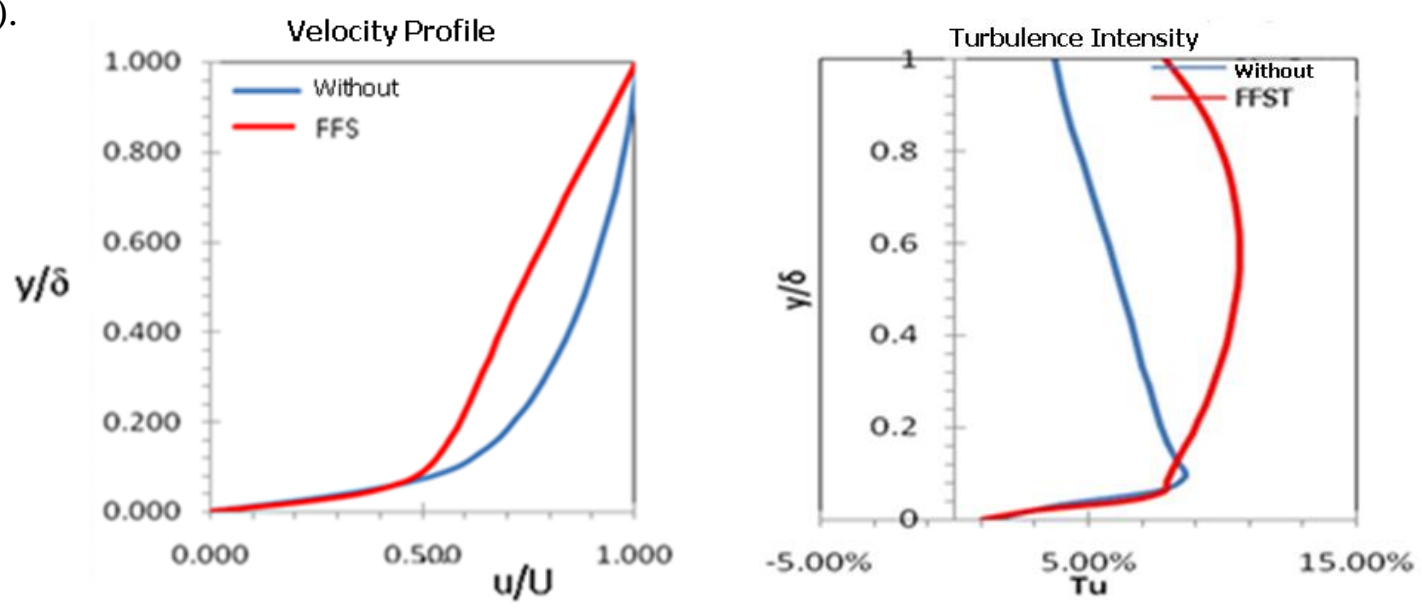

Fig.2 Velocity profile and turbulence intensity (without and with FFST)

A slight weakening of momentum flow near the end wall causes stagnation point on the leading edge has a higher position on the end wall. Fig. 3 shows the end position of the leading edge stagnation point between the flow with and without the addition of FFST.
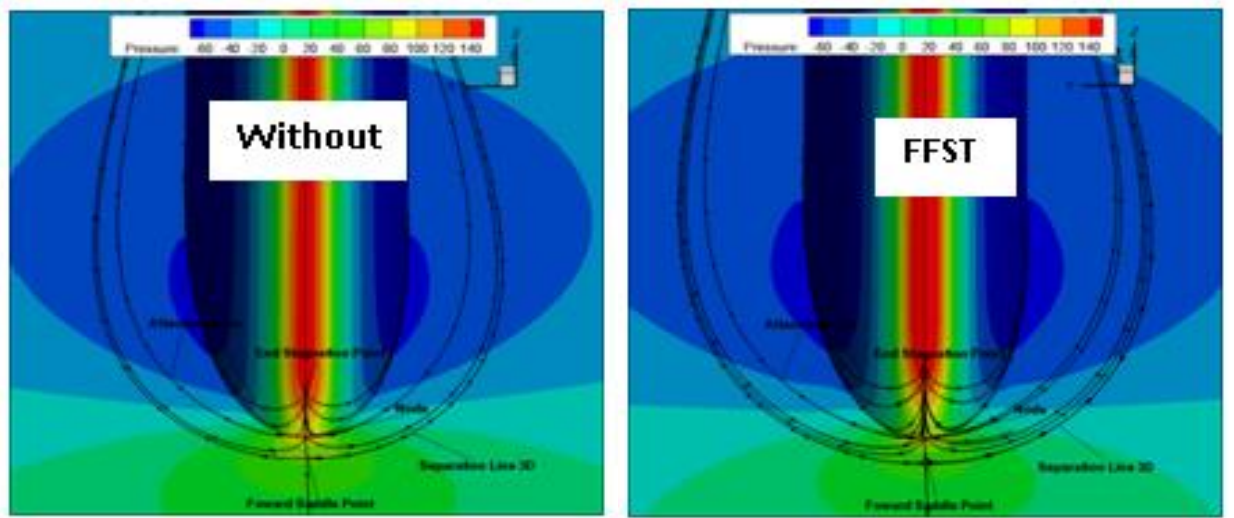

Fig.3 Comparation of end position of stagnation point, with FFST and without FFST

Additional FFST leads the formation of 3D separation line wider and far away from the body contour. Furthermore, turbulent intensity on the flow will gradually increase the velocity and momentum of flow. The flow has more ability to overcome adverse pressure gradient and friction effects. It delays the separation point and the corner wake at the juction is narrowed. As the result, the blockage and energy losses can be reduced. Path lines for the numerical simulations and experimental work using oil flow visualization (OFV) are presented in Figs. 4 and 5, respectively. It can be observed that the numerical and exprimental works give an identic flow structure. Therefore the numerical modeling will be used to reinforce and explain the detailed analysis of the flow. 

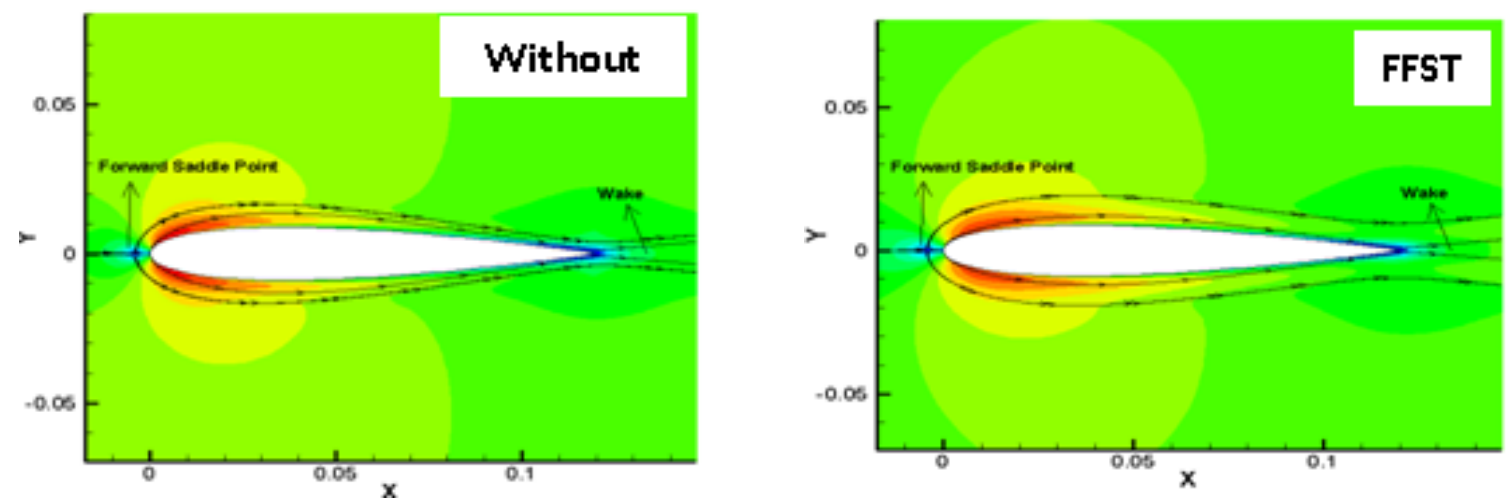

Fig.4 Comparation of Flow Structure without and with FFST at $\alpha=0^{0}$ (Numerik)
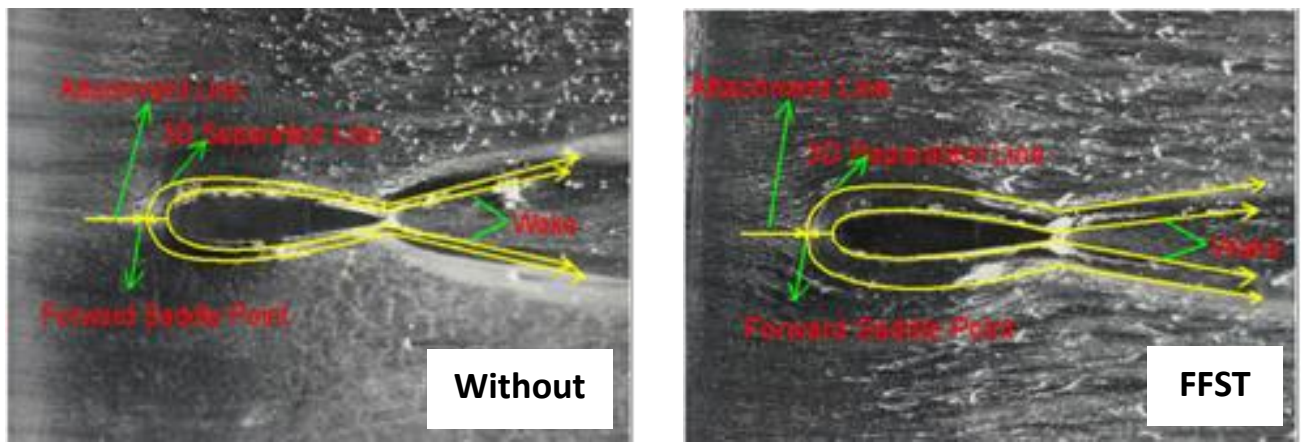

Fig.5 Comparation of Flow Structure without and with FFST at $\alpha=0^{0}$ (experiment)

OFV result in Fig. 5 shows that the form of stream tube (3d separation line) is initially open and away from the body. Due to a stronger turbulence intensity, attachment line on the upper side and lower side are able to control the horse shoe vortex formation and the flow can follow the contours of the body better than the case of without FFST. It is shown by the location of the separation point can be delayed and shape of fish tail on the trailing is slimmer. This is a new phenomenon 3D flow structure near the endwall.
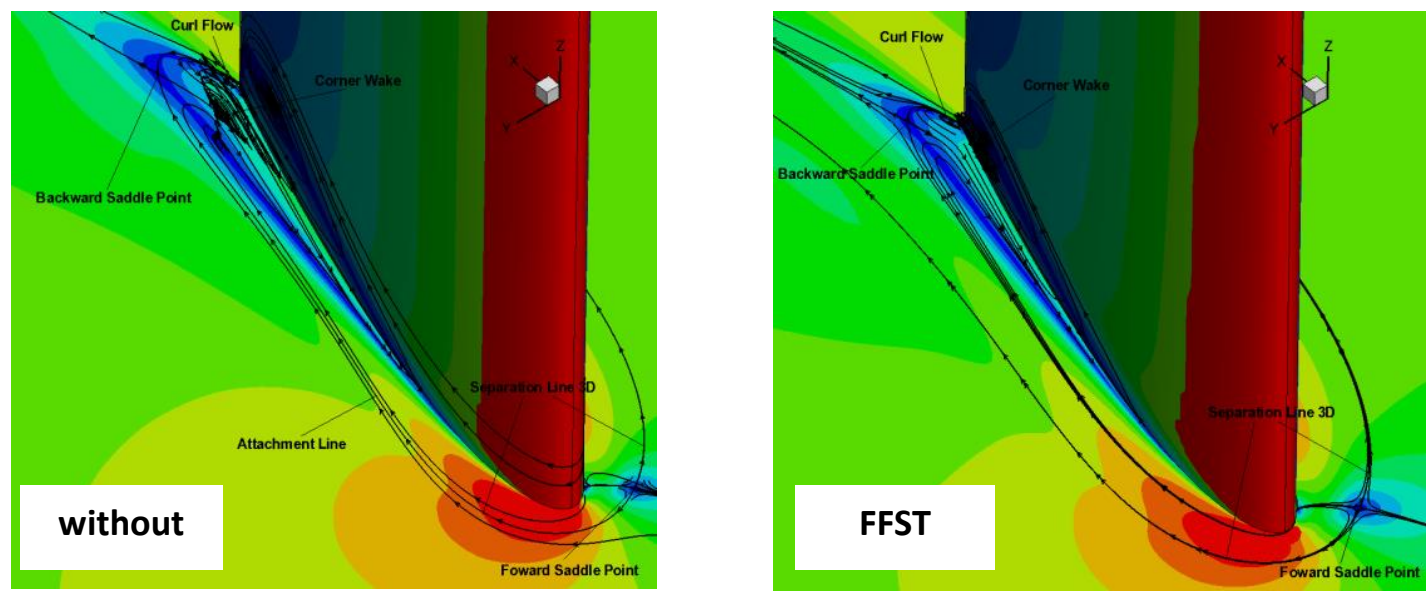

Fig. 6 Pathlines structure without and with FFST at $\alpha=12^{0}$

Flow structure (distribution of pressure and path lines) on the upper side on the $\alpha=12^{0}$ is shown in Fig.6. The addition of $\alpha$ causes the saddle point move towards to the lower side. As described by Mirmanto and Sasongko (2004). When the angle of attack is enlarged, the frontal area which faces an inlet free-stream becomes wider and contact-angle which normal to the flow 
direction deviates to lower side. This condition causes path of the lower-side to be shorter and the flow is decelerated due of effect of increasing the pressure.

Acceleration occurs on the upper-side, and flow from the lower side tends to move to the low energy region on the upper side due to the pressure difference near the trailing edge This flow is known as curl flow and causes backflow on the upper side and strengthen the adverse pressure gradient. On the upper side, the attachment line follows contour body and meet with curl flow, the meeting point is called as backward-saddle-point. That meeting creates two branching separation line, one moves downstream and the other along with a backflow generate curl flow, strengthen the adverse pressure gradient and finally create a stronger flow separation.

Due to the meeting of backflow with free-stream, the flow to move towards mid span and empties near the trailing edge. The phenomenon is called the corner-wake which cause blockage flow and energy loss. The position of the saddle point in the flow with the addition of FFST has more distance to the leading edge as well as a wider the shape of separation line 3D on upstream of leading edge than the flow without FFST. Because the flow has higher turbulence intensity, both the lower and upper sides can control the horseshoe vortex formation better than the configuration without FFST and flow is able to follow the contours of the body.

On the lower side, attachment line with higher turbulent intensity is capable of moving towards downstream in such a way that the intensity of curl flow be weaker. On the upper side, the attachment line has more capability to move independently to follow the body contour and join with the three-dimensional separation line as occured for the flow without FFST.

Attachments line that move follow the body contour then face the curl flow. However intensity of the curl flow is weak, so increasing of adverse pressure gradient due to the backflow is not significant. Therefore the corner wake and flow blockage as well as energy loss can be reduced. It is shown in Fig. 6 that the flow with the addition FFST only controls a narrower area.

Energy loss is presented in the form of pressure drop and velocity to the axial direction, so that the amount of energy loss can be represented by total pressure loss coefficient. Fig. 7 show the total pressure loss coefficient of numerical modeling results for endwall (without and with FFST) on the angle of attack $\alpha=0^{0}$ and $\alpha=12^{0}$. The other values for different angle of attack can be seen in Table 2.

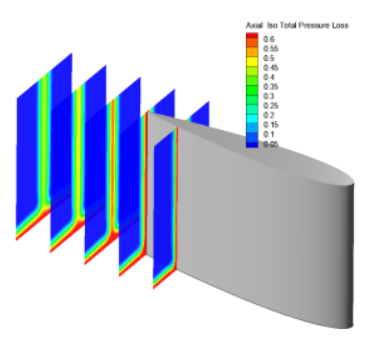

without $\alpha=0$

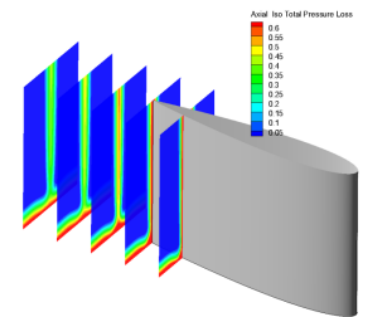

FFST $\alpha=0$
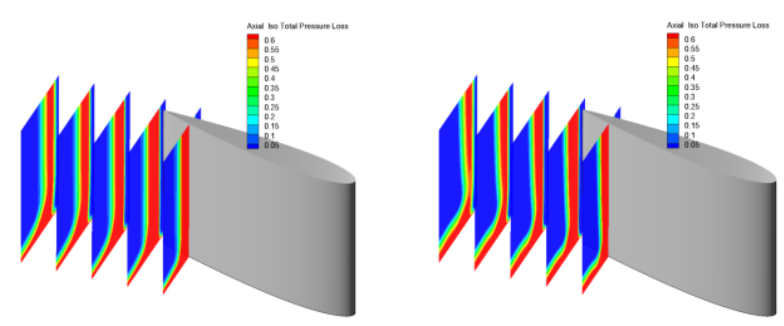

without $\alpha=12^{0}$

FFST $\alpha=12^{0}$

Figure 7 Contour of Iso Total Pressure Loss Coefficient at downstream

Table 2. Energy Loss Reduction

\begin{tabular}{||c|c|c|c|c|c||}
\hline \multirow{2}{*}{$\alpha$} & \multicolumn{2}{|c|}{$\begin{array}{c}\text { Total Pressure } \\
\text { Outlet }\end{array}$} & \multicolumn{3}{|c|}{ Energy Loss } \\
\cline { 2 - 6 } & $\begin{array}{c}\text { Flat } \\
\text { Plate }\end{array}$ & FFST & $\begin{array}{c}\text { Flat } \\
\text { Plate }\end{array}$ & FFST & Reduction \\
\hline 0 & 103.501 & 108.617 & $24.90 \%$ & $21.18 \%$ & $3.71 \%$ \\
4 & 103.384 & 108.687 & $24.98 \%$ & $21.13 \%$ & $3.85 \%$ \\
8 & 101.079 & 106.802 & $26.65 \%$ & $22.50 \%$ & $4.15 \%$ \\
12 & 95.268 & 100.139 & $30.87 \%$ & $27.34 \%$ & $3.53 \%$ \\
16 & 99.488 & 94.435 & $27.81 \%$ & $31.48 \%$ & $-3.67 \%$ \\
\hline
\end{tabular}




\section{Conclusions :}

Characteristics of the flow with the addition of FFST can be summarized as follows:

At $\alpha=\mathbf{0}^{\mathbf{0}}$ :

1. The end position of stagnation point on the leading edge is shifted away relative to the endwall.

2. Attachment line derived from the end branching of stagnation point, has a stronger turbulence intensity and controlling the horseshoe vortex formation, making it capable of pressing 3D separation line away from the body contour.

3. A higher turbulence intensity can reduce the corner wake and energy loss in the downstream area.

At $\alpha=0^{0}-12^{0}$

1. At higher $\alpha$, intensity of curl flow at the trailing edge becomes stronger, but can be attenuated by the attachment line on the lower side which has a stronger turbulence intensity.

2. On the upper side, the attachment line has higher turbulence intensity, so it is not easily induced by the horseshoe vortex. Attachment line trace closer to the body contour, making it easier to meet a curl flow, thus backflow and adverse pressure gradient can be attenuated. Furthermore, the corner wake becomes narrower and blockage as well as energy loss can be reduced.

\section{References}

[1]. Horlock,J.H, "Leakage and Secondary Flows in Compressor Cascades", Ministry of Tecnology Aeronautical Research Council Report and Memoranda No.3483, (1987).

[2]. Mirmanto, Sasongko, "Secondary Loss Reduction Through Addition of Leading Edge Fairing”. Jurnal Teknik Mesin ITS, Surabaya, (2009).

[3]. Schiliting Herman, translated by DR J Kestin," Boundary Layer Theory", $7^{\text {th }}$ edition, Mc GrawHill Book Company, (1979).

[4]. Djilali.N, Gratshore.I.S, "Turbulent flow around a bluff rectangular plate, Part1: Experimental investigation", ASME Trans. Fluid Eng. 11pp51-59, (1991).

[5]. Yaghoubi. M, Mahmoodi. S, "Experiment study of turbulent separated and reattached flow over a finite blunt plate, Ekperimental Thermal and Fluid Science 29-pp 105-112, (2004).

[6]. Suksangpanomrung. A, et.al, "Large-eddy simulation of separated flow over a bluff rectangular plate", Internasional journal of heat transfer fluid flow 21-655-663, (2002).

[7]. Saldana J.G.B, et.al, "Numeric simulation for the flow structures following a three dimensional horizontal forward-facing step channel", Revista mexcama de fisica53(2)pp 8795, (2007).

[8]. Sherry M. J, et.al, "Flow separation characterization of a forward facing step immersed in a turbulent boundary layer", Ekperimental Thermal and Fluid Science 5-pp 88-102, (2010).

[9]. Jones D.A. and Clarke D. B.,"Simulation of a Wing Body Junction Experiment using Fluent code, Defence science and technology organization (DSTO)-TR-1731, (2005). 